\title{
DESENVOLVIMENTO E CONFIRMAÇÃO DE VALIDADE E DE CONFIABILIDADE DO INSTRUMENTO DE COLETA DE DADOS SOBRE A FORMAÇÃO INICIAL DE PROFESSORES E TECNOLOGIAS EDUCACIONAIS
}

\author{
Mariana Haviaras \\ Herivelto Moreira $^{* *}$ \\ Claudia Beatriz Monte Jorge Martins ${ }^{* * *}$
}

Resumo: A elaboração de um instrumento de coleta de dados requer suporte teórico e critérios para verificar a validade e a confiabilidade. Para isso, esse artigo tem como objetivo apresentar o desenvolvimento de um instrumento de coleta de dados e o seu processo de confirmação: validade e confiabilidade. A motivação pela construção se deu a partir da fase inicial da tese da pesquisadora em que se percebeu a necessidade de apresentar aos pesquisadores da área educacional a importância da validação e da confiabilidade de instrumentos de coletas de dados em pesquisas científicas. $\mathrm{O}$ artigo foi elaborado por meio de um levantamento bibliográfico em livros e artigos publicados sobre o assunto e também com base no estudo-piloto da tese da pesquisadora. Conclui-se sugerindo que estudos na área educacional com vistas a perspectiva quantitativa sejam realizados e que os devidos cuidados sejam tomados, refletindo sempre que as pesquisas merecem sérias considerações e rigorosidade.

Palavras-chave: Instrumento de coleta de dados. Validade. Confiabilidade.

\section{Introdução}

O presente artigo tem como base o estudo-piloto de uma tese de doutorado sobre a formação inicial de professores para o uso de tecnologias educacionais. Para a elaboração da tese optou-se pelo levantamento quantitativo, uma vez que possibilita que a pesquisa seja aplicada a uma população ou amostra de uma população maior, sem desperdício de tempo, que dados numéricos sejam obtidos, mas também que o cruzamento de informações seja realizado por meio de pacotes estatísticos. Levantamentos quantitativos são utilizados na área educacional assim como em outros campos de estudos (ARTINO JUNIOR et al., 2014; ZHANG, 2000), especialmente estudos que focam a questão da tecnologia.

\footnotetext{
* Universidade Tecnológica Federal do Paraná - (UTFPR), Campus Curitiba, Curitiba - Paraná - Brasil. Doutoranda do Programa de Pós-Graduação em Tecnologia e Sociedade. E-mail: marianahaviaras@gmail.com.

** Universidade Tecnológica Federal do Paraná - (UTFPR), Campus Curitiba, Curitiba - Paraná - Brasil. Doutor e docente do Programa de Pós-Graduação em Tecnologia e Sociedade. E-mail: herivelto.moreira51@gmail.com.

*** Universidade Tecnológica Federal do Paraná - (UTFPR), Campus Curitiba, Curitiba - Paraná - Brasil. Doutora e docente da UTFPR. E-mail: claudiab@utfpr.edu.br.
} 


\section{\#tear}

Apesar de os questionários serem o principal instrumento de coleta de dados usado em levantamentos quantitativos (MARCONI; LAKATOS, 2011; MOREIRA; CALEFFE, 2006), constatou-se a não existência de um questionário específico que pudesse ser utilizado ou adaptado para o estudo sobre a formação inicial de professores em relação ao uso de tecnologias educacionais. Dessa forma, foi elaborado o instrumento de coleta de dados, intitulado Formação inicial de professores e tecnologias educacionais, a partir de uma revisão de literatura (BRITO; PURIFICAÇÃO, 2012; GARCÍA, 1999; KENSKI, 2015; SANCHO, 2007, entre outros) que aborda a temática de formação de professores e tecnologias e também de outros instrumentos já desenvolvidos e publicados (GRAVONSKI, 2013; LUZ, 2018; MARTINS, 2015; ROCHA, 2018).

Além disso, observou-se também que grande parte dos questionários não passa por um processo de validação e de confiabilidade, o que pode comprometer o rigor e a análise dos resultados obtidos. O referido questionário, Formação inicial de professores e tecnologias educacionais, passou pelo processo de validação e de confiabilidade, os quais estão descritos detalhamente nas próximas seções, em que foi avaliada a efetividade e a consistência interna do instrumento de coleta de dados, respectivamente.

Assim sendo, com este artigo objetiva-se apresentar o desenvolvimento do instrumento para coleta de dados, Formação inicial de professores e tecnologias educacionais, produzido para mensurar como os professores dos cursos de Pedagogia do município de Curitiba, estado do Paraná, utilizam as tecnologias educacionais na formação de futuros professores/pedagogos; bem como apresentar o processo de confirmação de validade e da confiabilidade desse instrumento de coleta de dados.

A importância desse artigo se dá pela necessidade de se utilizar as nomenclaturas e termos corretos e adequados a cada tipo de pesquisa, bem como os devidos critérios que devem ser utilizados para que o rigor seja observado na condução de estudos do tipo levantamento quantitativo com o uso de questionários.

Dessa forma, espera-se que o processo de validação do mencionado instrumento possa ser seguido e ampliado para pesquisa futuras e também que o questionário, Formação inicial de professores e tecnologias educacionais, possa ser aplicado em outros contextos educacionais. 
2 Estudos desenvolvidos sobre a temática formação inicial de professores e as tecnologias educacionais

$\mathrm{Na}$ fase inicial deste estudo, fez-se uma busca por teses e dissertações desenvolvidas na área de Formação inicial de professores e as tecnologias educacionais, a fim de se detectar a originalidade do trabalho.

Essa busca foi realizada nos sites de três importantes universidades brasileiras: Universidade de São Paulo (USP), Universidade Federal do Paraná (UFPR) e Pontifícia Universidade Católica do Paraná (PUCPR) e também no site da Coordenação de Aperfeiçoamento de Pessoal de Nível Superior (CAPES).

A busca por teses e dissertações se concentrou mais em instituições de ensino superior do estado do Paraná, pois essa região, mais especificamente Curitiba, é o foco principal do estudo.

Dessa compilação, foram encontrados 16 estudos que se relacionam à temática. Verificou-se que são desenvolvidas pesquisas na área educacional do tipo levantamento quantitativo, todavia, algumas apresentam uma denominação errônea, pois as classificam como pesquisas do tipo levantamento qualitativo, mas se utilizam de métodos quantitativos, o que as tornam pesquisas quantitativas ou pesquisas de métodos mistos, que se utilizam de instrumento de coleta de dados qualitativos e quantitativos.

Observou-se que não há pesquisas desse tipo realizadas em todas as instituições de ensino superior de Curitiba que visem detectar a formação inicial de professores e as tecnologias educacionais e, com isso, ressalta-se a originalidade do estudo.

Por fim, para este artigo, voltou-se o olhar novamente aos 16 estudos desenvolvidos com a intenção de observar se as teses e dissertações que se utilizam de questionários para a coleta de dados passaram por um processo de validação e confiabilidade do instrumento de coleta de dados. Dos 16 estudos, nove utilizaram o questionário como instrumento para a coleta de dados, mas observou-se que apenas uma das pesquisas passou pelo processo de validação e de confiabilidade.

Este artigo amplia o processo de confirmação de validade e de confiabilidade realizado por Silva (2015) ao descrever, nos tópicos seguintes, a fase da confirmação da validade de conteúdo e de aparência do instrumento de coleta de dados, fase essa que não foi encontrada mesmo no estudo acima referido. 


\title{
\#tear
}

Os demais estudos ${ }^{1}$, se passaram pelo processo de validação do instrumento de coleta de dados, não as descreveram detalhadamente em seu relatório final.

Um relato muito interessante em uma das pesquisas, a dissertação de Castro (2014, p. 49) pela Universidade Federal de Rondônia, ressalta que:

\begin{abstract}
A elaboração do questionário seguiu uma série de cuidados para formular as questões no que se refere: a ordem, a quantidade, a clareza e a precisão. Mesmo assim, foi necessário reaplicá-lo: ao tabular os dados, detectamos incoerências. $\mathrm{O}$ motivo de tal incoerência se deu devido a falta de leitura e/ou interpretação errônea do enunciado. Dessa forma, foi reformulado e reaplicado o questionário, procurando assim, a veracidade dos dados.
\end{abstract}

Essa é uma constatação muito importante e que vai ao encontro deste artigo quando se verifica a importância de realização do estudo-piloto e validação do instrumento de coleta de dados para que os dados sejam fidedignos, retratem a realidade pesquisada e não haja a necessidade de administrar novamente o o questionário à amostra ou população.

Assim, pretende-se apresentar o caminho trilhado até o momento na presente pesquisa e a importância de atender os rigores necessários para a elaboração de instrumentos de coleta de dados quantitativos, que estão descritos nos tópicos a seguir.

\section{A pesquisa quantitativa e o desenvolvimento de um instrumento para a coleta de dados}

Diversas são as vantagens a partir de uma pesquisa quantitativa. Para Moreira e Caleffe (2006, p. 73) esse tipo de pesquisa “explora as características e situações de que dados numéricos podem ser obtidos e faz uso da mensuração e estatísticas”. Além dessa utilidade, a partir de pesquisas quantitativas, é possível coletar dados com a população ou uma amostra da população sem que o tempo seja fator limitador, pois nesse tipo de pesquisa o pesquisador consegue coletar dados com amostras maiores em espaço menor de tempo.

A elaboração de questionários é um componente crucial, é a sua essência (KROSNICK; PRESSER, 2010). O processo de desenvolvimento requer atenção e cuidado para que todas as informações estejam claras ao respondente e também que sejam feitas todas as perguntas a que se deseja responder em um determinado estudo.

O instrumento de coleta de dados, Formação inicial de professores e tecnologias educacionais, foi elaborado a partir da revisão de literatura (BRITO; PURIFICAÇÃO, 2012; GARCÍA, 1999; KENSKI, 2015; SANCHO, 2007, entre outros) que aborda essa temática e

\footnotetext{
${ }^{1}$ Um dos estudos trouxe como nota de rodapé a descrição de que havia sido realizado um questionário piloto, mas não descreveu esse processo, somente apresentou a versão final do mesmo no apêndice do trabalho.
} 
também de outros instrumentos de pesquisas já desenvolvidas e publicadas (GRAVONSKI, 2013; LUZ, 2018; MARTINS, 2015; ROCHA, 2018).

Esse processo durou cerca de 2 meses, com início em março e término em abril de 2017, em que a pesquisadora e seu orientador discutiram em torno da temática e chegaram a um instrumento inicial. A primeira versão do questionário continha 125 itens divididos em seis blocos:

a) Bloco 1: Característica dos respondentes, 11 itens. Os itens desse bloco foram elaborados com o objetivo de caracterizar os respondentes.

b) Bloco 2: Percepção dos professores sobre as tecnologias educacionais, composto de uma escala do tipo Likert, que se utilizou de alternativas "discordo totalmente", "discordo", "nem concordo/nem discordo", "concordo" e "concordo totalmente", 34 itens. Nesse bloco foram construídas questões com a finalidade de detectar a percepção dos professores dos cursos de Pedagogia sobre as tecnologias educacionais.

c) Bloco 3: Estratégias metodológicas utilizadas pelos professores, composto de uma escala de frequência, que se utilizaram de alternativas "nunca", "às vezes", "frequentemente" e "sempre", 42 itens. O objetivo desse bloco era mensurar a periodicidade com que os professores utilizam diferentes encaminhamentos didáticos utilizando as tecnologias nas aulas e atividades com os alunos.

d) Bloco 4: Infraestrutura e incentivo institucional, composto pela escala dicotômica, que se utilizou das alternativas "sim" e "não", 19 itens. O objetivo era verificar a infraestrutura tecnológica disponível em termos de quantidade e qualidade para os professores nas instituições de ensino superior, entre outras questões.

e) Bloco 5: Formação inicial e continuada para a utilização de tecnologias educacionais, composto de uma escala de frequência, que se utilizaram de alternativas "nunca", "às vezes", "frequentemente" e "sempre", 14 itens. O objetivo era mensurar como ocorre o processo de formação inicial e continuada dos professores dos cursos de Pedagogia com o uso das tecnologias.

f) Bloco 6: Grau de importância de formação sobre tecnologias educacionais, composto pela escala dicotômica, que se utilizou das alternativas "sim" e "não", 5 itens. O último bloco de questões foi proposto no sentido de mensurar o grau de importância que os professores dos cursos de Pedagogia atribuem à oferta de cursos de formação de professores voltados às tecnologias educacionais. 


\section{\#tear}

Para Moreira e Caleffe (2006, p. 113) "o uso de respostas em escalas possibilita ao pesquisador verificar a frequência de respostas para cada nível da escala”. Portanto, no caso do presente instrumento de coleta de dados, foram utilizadas a escala de Likert, que se utiliza dos níveis de concordância das afirmações propostas; a escala de frequência, que quantifica a periodicidade dos fatos destacados; e a escala dicotômica, que mensura se a afirmação acontece ou não.

A partir da elaboração dos itens das escalas, o passo seguinte foi verificar a validade de aparência e de conteúdo do instrumento de coleta de dados, que serão descritos a seguir.

\section{Validação da aparência e de conteúdo do instrumento de coleta de dados}

De acordo com Bittencourt et al. (2011) são poucos os trabalhos específicos sobre validação de instrumentos na área de educação no Brasil. Os autores enfatizam que a validação é um fator determinante na escolha e/ ou aplicação de um instrumento de medida. Ela é mensurada pela extensão ou grau em que o dado representa o conceito que o instrumento se propõe a medir (BITTENCOURT et al., 2011). Richardson (1999, p. 174) afirma que a validade de um questionário "é a característica de maior importância para avaliar sua efetividade". Ou seja, um questionário é válido se ele mede o que se deseja.

Dentre os métodos usados pelos pesquisadores para obtenção da validade de uma medida estão a validade de construto, a validade de critério, a validade de conteúdo e a validade de aparência (HOWELL et al., 2017; PASQUALI, 2009). Para o presente estudo foram usadas a validade de aparência e a validade de conteúdo.

Validade de aparência diz respeito à aparência de uma medida, ou seja, se ela parece uma forma razoável de se obter as informações pretendidas, se ela parece funcionar de maneira confiável, se parece que foi bem concebida. A validade de aparência não depende de teorias estabelecidas para ter suporte (HOWELL et al., 2017).

Validade de conteúdo "indica até que ponto o teste representa bem o tipo de situação estudada, e assim pode ser considerado válido como amostra representativa [...].” (GIL, 1999, p. 151). O conteúdo de um instrumento, ou seja, os itens e perguntas são amostras de situações diversas. A validade de conteúdo vai determinar o grau com que esses itens e perguntas representam determinadas situações (RICHARDSON, 1999).

A fim de verificar a validade de aparência e de conteúdo de um instrumento de coleta de dados, no caso o questionário, Gil (2008) ressalta que existem dois procedimentos frequentemente utilizados: (1) a opinião de um júri e (2) os grupos conhecidos. Na opinião de 


\section{\#tear}

um júri "a validade de uma escala é confirmada a partir da opinião de um grupo de pessoas tidas como especialistas no campo dentro do qual se aplica a escala" (GIL, 2008, p. 138). Já no procedimento de grupos conhecidos "a validade é obtida a partir das opiniões ou atitudes manifestadas por grupos opostos" (GIL, 2008, p. 138).

Para este estudo foi realizada a opinião de um júri, pois não havia o tempo para confrontação de dados entre grupos conhecidos, conforme a segunda proposta sugerida por Gil (2008).

Assim, o questionário foi submetido a validação de aparência e de conteúdo de três doutores que possuem reconhecimento científico na área de estudo da pesquisadora. A seleção deu-se pela vasta experiência na temática estudada e pelas publicações dos doutores na área.

Nessa etapa os doutores fizeram observações e sugestões de melhorias no conteúdo e na aparência do instrumento de coleta de dados. Com base nos apontamentos feitos pelos doutores foram realizados os ajustes necessários e o questionário foi readequado.

Dentre as sugestões feitas pelos especialistas optou-se por utilizar apenas a nomenclatura tecnologias educacionais em todo o instrumento, visto ser essa terminologia adotada na revisão de literatura; a divisão em dois blocos sobre a formação de professores: formação inicial de professores e formação continuada de professores, devido a especificidade de cada fase de formação; também foram suprimidos alguns itens que estavam semelhantes; e o layout do bloco de questões do perfil demográfico foi ajustado.

Após as modificações sugeridas pelos doutores, chegou-se a versão do questionário para ser aplicada no estudo-piloto, que contemplou 112 itens divididos em sete blocos, a seguir destacados:

a) Bloco 1: Característica dos respondentes, 10 itens.

b) Bloco 2: Percepção dos professores, que trabalham na formação dos futuros pedagogos, sobre as tecnologias educacionais, 29 itens.

c) Bloco 3: Estratégias metodológicas utilizadas pelos professores formadores de futuros pedagogos, 37 itens.

d) Bloco 4: Infraestrutura e incentivo institucional, 16 itens.

e) Bloco 5: Formação inicial para a utilização de tecnologias educacionais, 6 itens.

f) Bloco 6: Formação continuada para a utilização de tecnologias educacionais, 11 itens.

g) Bloco 7: Grau de importância de formação sobre tecnologias educacionais, 3 itens. 


\section{\#tear}

As escalas foram mantidas, portanto, o bloco 2 ficou composto pela escala do tipo Likert. Os blocos 3 e 5 ficaram compostos pelas escalas de frequência. Já o bloco 4 ficou composto pela escala dicotômica.

$\mathrm{Na}$ sequência, o questionário foi submetido ao estudo-piloto e ao teste de confiabilidade e de fidedignidade, conforme detalhes descritos a seguir.

\section{0 estudo-piloto}

O estudo-piloto é uma fase da pesquisa em que o(a) pesquisador(a) aplica o instrumento de coleta de dados a uma amostra considerável da população a fim de testá-lo previamente. Para Moreira e Caleffe (2006, p. 27) o objetivo do estudo-piloto "é simular a situação real tão fielmente quanto possível e usar uma população semelhante, selecionando a amostra da mesma maneira, mas com tamanho menor, e estabelecendo as mesmas condições para a administração e para as respostas”.

A população para este estudo é composta de 220 professores dos cursos de Pedagogia das universidades privadas do município de Curitiba, estado do Paraná. O questionário foi aplicado a uma amostra da população, no caso, 28 professores. Para Gil (2008) a aplicação do instrumento de coleta de dados para 10 a 20 respondentes já é um número satisfatório, contanto que os respondentes façam parte da população de onde a amostra é retirada.

Essa fase de aplicação do estudo-piloto durou cerca de 3 meses e iniciou em julho de 2017 com finalização em setembro de 2017. Cada um dos participantes levou em torno de 15 minutos para responder o questionário.

O instrumento continha linhas em branco ao final de cada bloco de questões. Essas linhas foram deixadas para que os respondentes pudessem incluir alternativas que consideravam relevantes para o estudo, bem como opiniões sobre o preenchimento do instrumento. Porém, apesar de essa possibilidade ser disponibilizada, não foram feitos comentários no questionário.

$\mathrm{Na}$ sequência da aplicação do questionário à amostra, os dados coletados e organizados no software Statistica, versão 13.3. Nesse momento, iniciou-se a fase de análise estatística preliminar com a intenção de testar a confiabilidade do questionário, fase essa que será apresentada a seguir. 


\section{$6 \mathrm{O}$ teste de confiabilidade do instrumento de coleta de dados}

Para testar a confiabilidade de um instrumento de coleta de dados existem alguns testes que podem ser utilizados. No caso do estudo-piloto realizado neste estudo, foi utilizado o teste denominado Alfa $(\alpha)$ de Cronbach.

O teste Alfa $(\alpha)$ de Cronbach recebe esse nome, pois no ano de 1951 o pesquisador Cronbach publicou um estudo em que abordava questões sobre estimação da consistência interna de uma escala em um questionário e proposições de autores para o cálculo dessa consistência. No estudo, Cronbach sugeriu uma estimativa de consistência interna a partir das variâncias dos itens e dos totais do teste por sujeito, o que ficou conhecido como "alfa" de Cronbach (MAROCO; GARCIA-MARQUES, 2006).

O objetivo do teste Alfa $(\alpha)$ de Cronbach é verificar se há aderência às questões do questionário, se os itens apresentam clareza e se há conexão entre os blocos de questões e também entre todas elas.

Maroco e Garcia-Marques (2006) destacam que quanto mais a escala do teste Alfa $(\alpha)$ de Cronbach se aproximar de 1, mais confiável e fidedigno será o questionário. Para que o instrumento de coleta de dados seja confiável deve obter, no mínimo, 0,70 no teste, mas os autores ressaltam que nas pesquisas sociais o número 0,60 é aceito, devido às especificidades realizadas nas pesquisas dessas áreas.

Os resultados obtidos com as escalas do instrumento utilizado nesse estudo foram maiores que 0,80 (Tabela 1), tanto para cada escala como para a consistência entre todas as questões. Portanto, o resultado indica que o instrumento de coleta de dados é confiável e suas questões são fidedignas, o que implicará em uma análise de dados confiável após a pesquisa ser aplicada a toda a população.

Tabela 1 - Resultados do teste Alfa de Cronbach

\begin{tabular}{cccc}
\hline Escala & Itens & $\begin{array}{c}\text { Alfa de } \\
\text { Cronbach }\end{array}$ & $\begin{array}{c}\text { Alfa de } \\
\text { Cronbach }\end{array}$ \\
\hline Bloco 2 & 29 & 0,844 & \\
Bloco 3 & 37 & 0,918 & \\
Bloco 4 & 16 & 0,860 & 0,922 \\
Bloco 5 & 6 & 0,877 & \\
Bloco 6 & 11 & 0,849 & \\
\hline
\end{tabular}

Fonte: Autora (2018)

Após a análise dos resultados do estudo-piloto foram feitas algumas alterações, mas não foi feita uma segunda aplicação do instrumento uma vez que as mudanças não foram 


\section{\#tear}

consideradas relevantes a ponto de alterar drasticamente o estudo real. Após estabelecer a confiabilidade do instrumento, chegou-se a versão final para ser aplicada a uma amostra maior.

7 Versão final do instrumento de coleta de dados: Formação inicial de professores e tecnologias educacionais

Após a fase de teste de verificação da validade e da confiabilidade, o instrumento (ver Apêndice A) passou a ter um total de 109 variáveis ou alternativas, distribuídas em 7 blocos:

a) Bloco 1: Características dos respondentes, 10 variáveis.

b) Bloco 2: Percepção sobre as tecnologias educacionais, 29 variáveis .

c) Bloco 3: Estratégias metodológicas de ensino, 37 variáveis.

d) Bloco 4: Infraestrutura e incentivo institucional, 16 variáveis.

e) Bloco 5: Formação inicial para a utilização de tecnologias educacionais, 6 variáveis.

f) Bloco 6: Formação continuada para a utilização de tecnologias educacionais, 8 variáveis.

g) Bloco 7: Grau de importância de formação sobre tecnologias educacionais, 3 variáveis.

Ou seja, de um total de 125 itens que foram propostos inicialmente para o instrumento, ficou 109 variáveis em sua versão final após todo o processo de confirmação da validade e da confiabilidade do instrumento de coleta de dados.

Como pôde-se observar ao longo deste artigo foi trilhado um caminho para a elaboração, confirmação da validade e da confiabilidade do instrumento de coleta de dados, Formação inicial de professores e tecnologias educacionais, o qual está contemplado na Figura 1: 


\section{\#tear \\ Revista de Educação, Ciência e Tecnologia}

Figura 1 - Fluxograma do processo de desenvolvimento do instrumento de coleta de dados

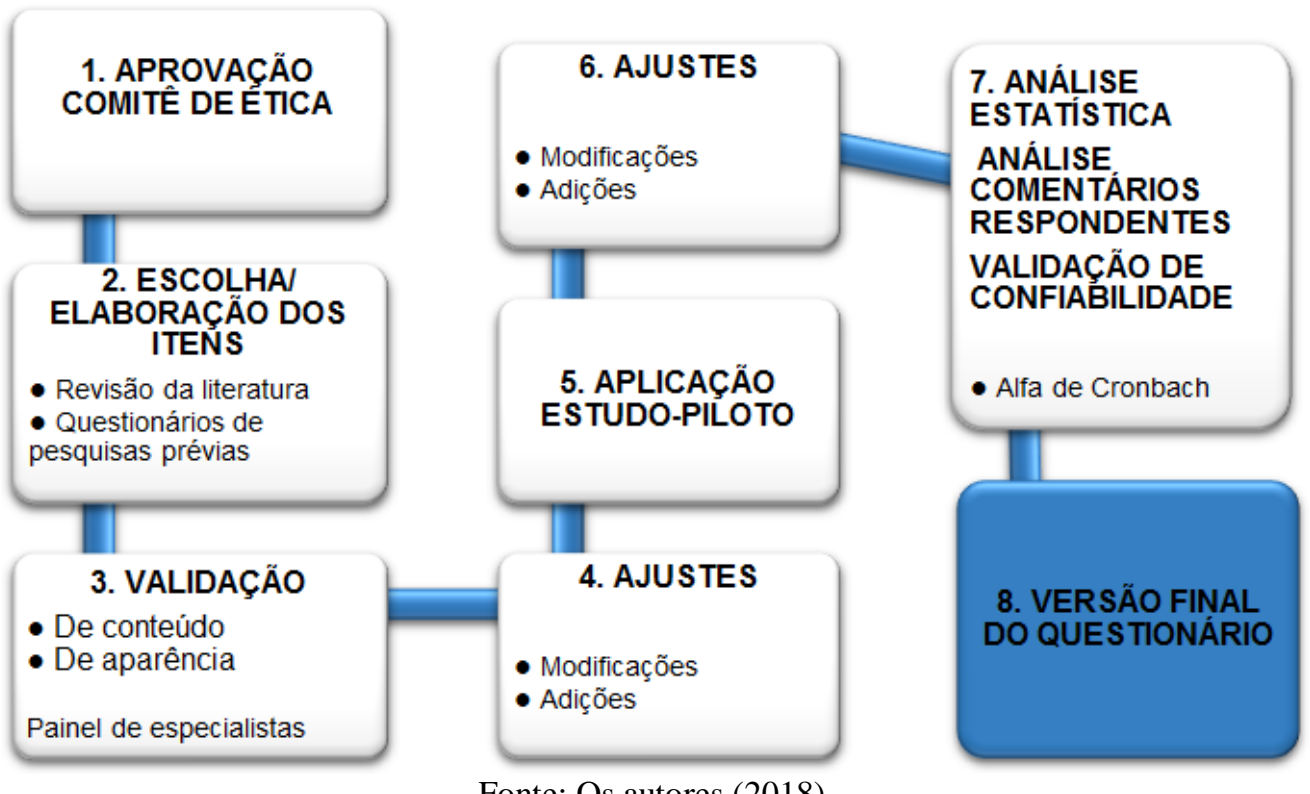

Fonte: Os autores (2018)

A Figura 1 resume, portanto, o processo percorrido para a elaboração, validação e confiabilidade do instrumento de coleta de dados, Formação inicial de professores e tecnologias educacionais, e traz em seu item 1 uma fase de extrema importância para as pesquisas científicas envolvendo seres humanos, a aprovação do Comitê de Ética.

Toda pesquisa que envolve seres humanos deve, necessariamente, passar previamente pela aprovação do Comitê de Ética da instituição a que se está desenvolvendo o estudo. Assim, a pesquisa que este artigo está descrevendo foi aprovada pelo Comitê de Ética da UTFPR e está protocolada na Plataforma Brasil, sendo essa a etapa inicial de toda pesquisa. As demais fases foram discorridas ao longo deste artigo.

\section{Considerações finais}

O objetivo deste artigo foi apresentar o desenvolvimento do instrumento para coleta de dados, intitulado Formação inicial de professores e tecnologias educacionais, produzido para mensurar como os professores dos cursos de Pedagogia do município de Curitiba, estado do Paraná, utilizam as tecnologias educacionais na formação de futuros professores/pedagogos; bem como apresentar o processo de confirmação de validade e da confiabilidade do referido instrumento de coleta de dados.

A elaboração do instrumento, Formação inicial de professores e tecnologias educacionais, foi favorecida a partir de questionários já validados (GRAVONSKI, 2013; LUZ, 2018; MARTINS, 2015; ROCHA, 2018) e utilizados em pesquisas de doutorado, e 


\section{\#tear}

também a partir da revisão de literatura que aborda a temática de formação de professores e tecnologias com a consolidação de autores da área (BRITO; PURIFICAÇÃO, 2012; GARCÍA, 1999; KENSKI, 2015; SANCHO, 2007, entre outros). Com isso, foi possível a sistematização de um questionário especificamente voltado a referida temática, o qual, até o momento, pelas pesquisas mencionadas, não existia.

Na sequência, o processo de validação do conteúdo e da aparência do instrumento de coleta de dados, Formação inicial de professores e tecnologias educacionais, foi essencial à medida que trouxe diferentes olhares ao questionário de juízes, doutores, com vasta experiência na área. A fase de validação (do conteúdo e da aparência) dos questionários é de extrema importância para a análise de sua efetividade e comprovação se, de fato, o instrumento mede o que pretende avaliar.

Após essa fase, a aplicação do estudo-piloto testou o instrumento com uma amostra da população, o que foi importante para identificar o que ainda necessitava ser ajustado e também o que estava em acordo. Por meio do estudo-piloto, portanto, é possível detectar falhas e corrigir em tempo o instrumento de coleta de dados, antes de ser aplicado à população.

Já com a análise dos dados obtidos no estudo-piloto, foi possível verificar a consistência interna das questões por meio do teste Alfa $(\alpha)$ de Cronbach, em que se detectou a confiabilidade dos itens que compunham o instrumento. Nesse sentido, essa etapa é primordial para a comprovação da veracidade do questionário.

Com o desenvolvimento do questionário, Formação inicial de professores e tecnologias educacionais, espera-se que ele possa ser utilizado em pesquisas futuras e, se necessário, ajustes e alterações sejam realizados, conforme o contexto a ser investigado. Almeja-se também que as etapas de validação e de confiabilidade do instrumento possam ser replicadas e sirvam como base para a construção de outros questionários e até mesmo complementadas em pesquisas vindouras de outros estudantes.

Assim sendo, conclui-se este artigo apontando a importância de se realizar pesquisas quantitativas, em que se possam ter dados numéricos e estatísticos e que os cruzamentos desses dados possam ser realizados. Também é crucial que as pesquisas quantitativas recebam a denominação adequada em trabalhos acadêmicos e que as fases de desenvolvimento e critérios de validação de instrumentos de coletas de dados sejam seguidas para que se obtenham resultados confiáveis. 


\title{
DEVELOPMENT AND CONFIRMATION OF THE VALIDITY AND RELIABILITY OF THE DATA COLLECTION INSTRUMENT ON INITIAL TEACHER TRAINING AND EDUCATIONAL TECHNOLOGIES
}

\begin{abstract}
The creation of a data collection instrument requires theoretical support and criteria so that the validity and reliability of the instrument can be checked. The present article aims to present the development of a questionnaire, as well as its process of validity and reliability. The motivation behind it came from the initial phase of the researcher's thesis. It was noticed the need to present to novice researchers in the educational area the importance of validation and reliability of data collection instruments. The article was based on a bibliographical survey of books and articles on the subject and also on the pilot study of the researcher's thesis. The article concludes by suggesting that other studies in the educational area with a view to a quantitative perspective should be carried out and that the necessary precautions should be taken, always reflecting that research, no matter its nature, deserves serious consideration and rigor.
\end{abstract}

Keywords: Data collection instrument. Validity. Reliability.

\section{Referências}

ARTINO JUNIOR, A.R. et al. Developing questionnaires for educational research: AMEE Guide No. 87. Medical Teacher, [s.1.], v. 36, n. 6, p.463-474, 24 mar. 2014.

BITTENCOURT, H.R. et al. Desenvolvimento e validação de um instrumento para avaliação de disciplinas na educação superior. Estudos em Avaliação Educacional, São Paulo, v. 22, n. 48, p.91-114, jan./abr. 2011. Disponível em:

<http://www.fcc.org.br/pesquisa/publicacoes/eae/arquivos/1630/1630.pdf > . Acesso em: 10 jan. 2018.

BRITO, G.S.; PURIFICAÇÃO, I. Educação e novas tecnologias: um repensar. 2. ed. Curitiba: InterSaberes, 2012. 143 p.

CASTRO, A.N. Formação inicial: A contribuição para o uso pedagógico das TIC na Educação Básica - Um estudo no curso de Pedagogia da UFMT/Campus Cuiabá. 2014. 114 f. Dissertação (Mestrado em Educação) - Universidade Federal de Rondônia, Porto Velho, 2014.

GARCÍA, C.M. Formação de professores para uma mudança educativa. Porto (Portugal): Porto Editora, 1999. 272 p.

GIL, A.C. Métodos e técnicas de pesquisa social. 5. ed. São Paulo: Atlas S. A., 1999. 206 p. 5. ed. São Paulo: Atlas S. A., 2008. 200 p.

GRAVONSKI, I.R. O desafio de formar formadores na e para a educação tecnológica: o método misto de pesquisa para análise dos saberes e da aprendizagem docente no contexto das Tecnologias de Informação e Comunicação. 2013. 283 f. Tese (Doutorado em Tecnologia) - Universidade Tecnológica Federal do Paraná, Curitiba, 2013. 
HOWELL, J. et al. Reliability and Validity. Writing@CSU. Colorado State University. Disponível em: <https://writing.colostate.edu/guides/page.cfm?pageid=1388\&guideid=66> . Acesso em: 17 jan.2017.

KENSKI, V.M. Tecnologias também servem para fazer educação. In: Educação e Tecnologias: O novo ritmo da educação. Campinas: Papirus, 2015. 144 p.

KROSNICK, J.A.; PRESSER, S. Question and Questionnaire Design. In: MARSDEN, P.V.; WRIGHT, J.D. (Ed.). Handbook of Survey Research. 2. ed. Bingley: Emerald Group Publishing Limited, 2010. Cap. 9. p. 263-314.

LUZ, S.V. A utilização de estratégias de ensino e de avaliação na formação de engenheiros: um estudo de métodos mistos. 2018. 264 f. Tese (Doutorado em Tecnologia e Sociedade) - Universidade Tecnológica Federal do Paraná, Curitiba, 2018.

MARCONI, M.A.; LAKATOS, E. M. Técnicas de pesquisa. 7. ed. São Paulo: Atlas S. A., 2011. 277 p.

MAROCO, J.; GARCIA-MARQUES, T. Qual a fiabilidade do Alfa de Cronbach? Questões antigas e soluções modernas? Laboratório de Psicologia: Instituto Superior de Psicologia Aplicada, Portugal, n. 4, p. 65-90, jul. 2006.

MARTINS, C. B. M. J. A Integração da Tecnologia nos Cursos de Licenciatura em Letras no Estado do Paraná a partir da Perspectiva dos Professores: um estudo de métodos mistos. 2015. 404 f. Tese (Doutorado em Tecnologia) - Universidade Tecnológica Federal do Paraná, Curitiba, 2015.

MOREIRA, H.; CALEFFE, L. G. Metodologia da pesquisa para o professor pesquisador. 1. ed. Rio de Janeiro: DP\&A, 2006. 245 p.

PASQUALI, L. Psicometria. Revista da Escola de Enfermagem da USP, São Paulo, v. 43, p.992-999, dez. 2009. Disponível em:

$<$ http://www.scielo.br/scielo.php?script=sci_arttext\&pid=S0080-

62342009000500002\&lng=en\&nrm=iso\&tlng=pt >. Acesso em: 10 jan. 2018.

RICHARDSON, R.J. Pesquisa social: métodos e técnicas. 3. ed. São Paulo: Atlas S. A., 1999.

ROCHA, R.F.Z. A tecnologia no processo ensino-aprendizagem na percepção dos professores das escolas de tempo integral da rede pública municipal de Curitiba. 2018. 328 f. Tese (Doutorado em Tecnologia e Sociedade) - Universidade Tecnológica Federal do Paraná, Curitiba, 2018.

SANCHO, J. M. De Tecnologias da Informação e Comunicação a Recursos Educativos. In: SANCHO, J.M.; HERNÁNDEZ, F. (Orgs.). Tecnologias para transformar a educação. Porto Alegre: Artmed, 2007. 198 p.

SILVA, F. C. M. Formação de professores e as tecnologias digitais na percepção dos professores e alunos do curso de Pedagogia da Universidade Federal do Ceará. 2015. 153 


\section{\#tear \\ Revista de Educação, Ciência e Tecnologia}

f. Dissertação (Mestrado em Políticas Públicas e Gestão da Educação Superior) Universidade Federal do Ceará, Fortaleza, 2015.

ZHANG, Y. Using the Internet for survey research: A case study. Journal of the American Society for Information Science, [s.1.], v. 51, n. 1, p.57-68, 21 jan. 2000. Wiley-Blackwell.

Apêndice A: Questionário - Formação inicial de professores e tecnologias educacionais

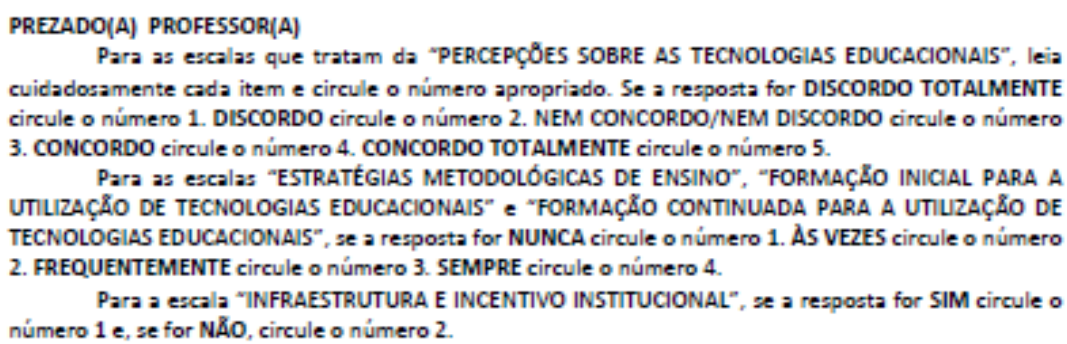

Para as eacalas que tratam da "PERCEPCOES SOBRE AS TECNOLOGIAS EDUCACIONAIS", leia cuidadosamente cada item e circule o número apropriado. Se a resposta for DISCORDO TOTALMENTE circule o número 1. DISCORDO circule o número 2. NEM CONCORDO/NEM DISCORDO circule o número 3. CONCORDO circule o número 4. CONCORDO TOTALMENTE circule o número 5.

Para as escalas "ESTRATÉGIAS METOdOLógICAS DE ENSINO", "FORMAÇăo INICIAL PARA A

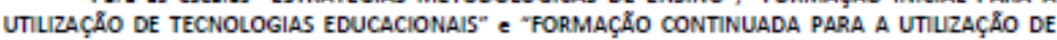
TECNOLOGIAS EDUCACIONAIS", se a resposta for NUNCA circule o número 1. AS VEZES circule o número 2. FREQUENTEMENTE circule o número 3. SEMPRE circule o número 4.

Para a escala "INFRAESTRUTURA E INCENTIVO INSTITUCIONAL", $x$ a resposta for SIM circule o número 1 e, se for $N A ̊ O O$, circule o número 2.

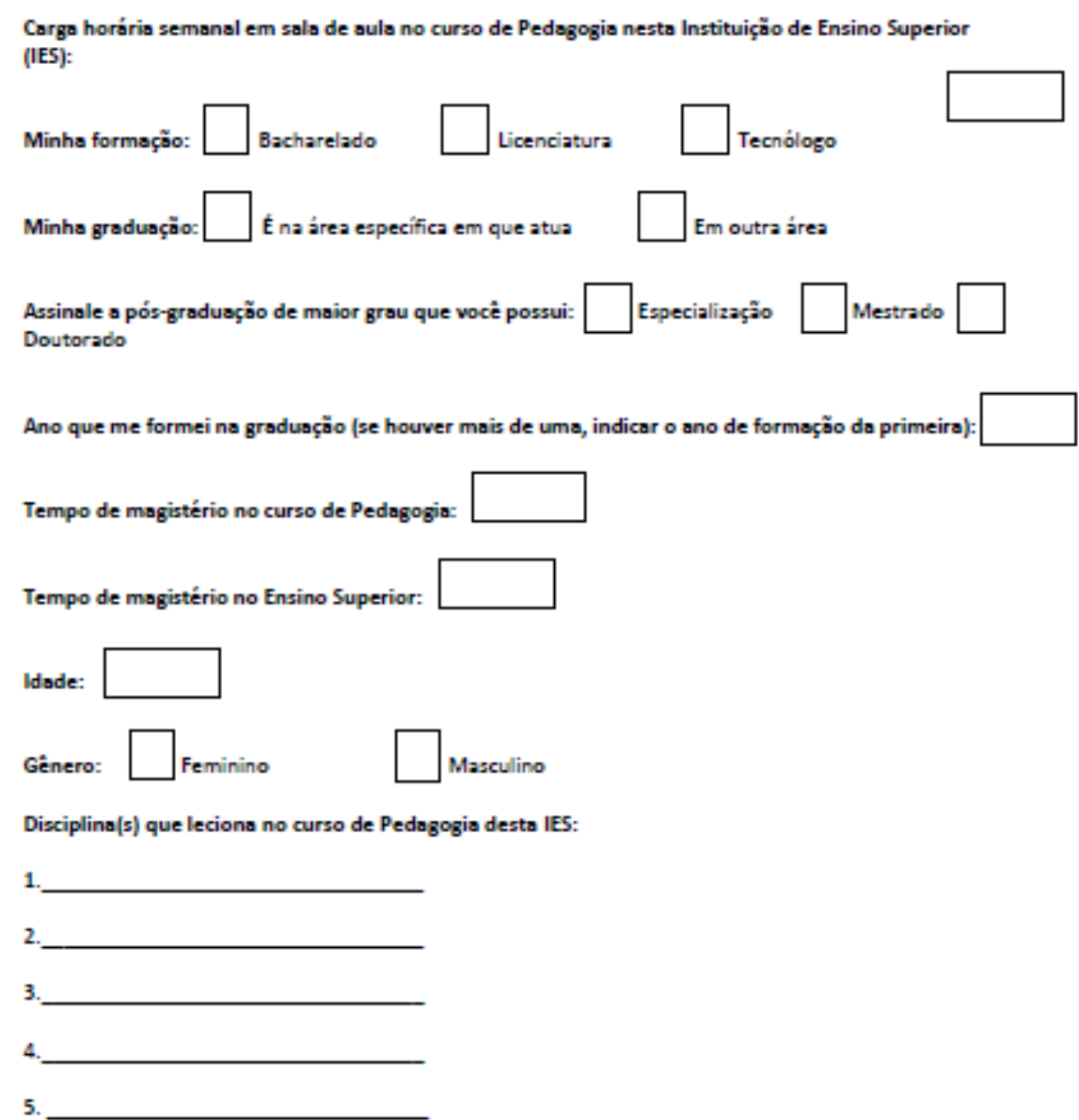


Assinale as suas percepções enquanto professor da graduação em Pedagogia:

A utilização de tecnologias educacionais para fins pedagógicos contribui para o processo ensinoaprendizagem dos futuros pedagogos.

A utilização de tecnologias educacionais contribui para a participação ativa dos alunos nas aulas.

A utilização de tecnologias educacionais torna a sala de aula um espaço educativo mais atrativo e motivante.

A utilização de tecnologias educacionais amplia a possibilidade de realização de experiências em grupo em sala de aula.

As tecnologias educacionais melhoram a relação interpessoal entre professor e aluno.

As tecnologias educacionais proporcionam maior variedade de estratégias metodológicas para as aulas.

As tecnologias educacionais apresentam-se como um recurso para auxiliar no desenvolvimento das aulas.

As tecnologias educacionais contribuem para ampliar o conhecimento trabalhado em sala de aula.

As tecnologias educacionais dificultam a ação do professor para trabalhar determinados conteúdos.

As tecnologias educacionais permitem que os alunos se tornem mais autónomos no seu processo de aprendizazem.

As tecnologias educacionais facilitam o trabalho do professor.

As tecnologias educacionais facilitam as atividades dos alunos.

As tecnologias educacionais oportunizam o trabal ho com metodologias ativas.

As tecnologias educacionais servem apenas para substituir o quadro e jiz.

As tecnologias educacionais propiciam atender os diferentes estilos de aprendizagem.

As tecnologias educacionais inovam o processo ensino-aprendizagem.

As tecnologias educacionais devem ser utilizadas apenas nos momentos da aula de informática.

O futuro pedagogo deve dominar as novas linguagens típicas de tecnologias educacionais utilizadas em sala de aula.

Tenho facilidade na utilização de tecnologias educacionais em sala de aula.

Tenho habilidades para trabal har com diferentes tecnologias educacionais.

Disponho de tempo suficiente para incorporar as tecnologias educacionais em minhas aulas.

Tenho receio em utilizar as tecnologias educacionais em minhas aulas.

Os meus alunos são mais preparados do que eu na manipulaçăo de tecnologias educacionais.

Sinto dificuldades para escolher a tecnologia educacional que melhor contribua para a aprendizagem do conteúdo da diaciplina que ministro.

Me considero capacitado para utilizar as tecnologias educacionais em sala de aula.

Com as minhas aulas, os futuros pedagogos estão aptos para utilizar tecnologias educacionais em

suas vindouras salas de aula.

A IES em que ministro aulas eatá equipada para a utilização de tecnologias educacionais.

O curso de graduação em Pedagogia da IES em que ministro aulas está preparando os alunos para trabalhar com as tecnologias educacionais.

A formação inicial relacionada à temática "Educação e Tecnologias" prepara o futuro pedagogo

para atuar na Sociedade da Informação e Comunicação.

\begin{tabular}{|l|l|l|l|l|l|}
\hline 1 & 2 & 3 & 4 & 5 \\
\hline 1 & 2 & 3 & 4 & 5 \\
\hline 1 & 2 & 3 & 4 & 5 \\
\hline 1 & 2 & 3 & 4 & 5 \\
\hline 1 & 2 & 3 & 4 & 5 \\
\hline & 2 & 3 & 4 & 5 \\
\hline
\end{tabular}

\begin{tabular}{|l|l|l|l|l|}
1 & 2 & 3 & 4 & 5 \\
\hline 1 & 2 & 3 & 4 & 5
\end{tabular}

\begin{tabular}{|l|l|l|l|l|}
1 & 2 & 3 & 4 & 5 \\
\hline
\end{tabular}

\begin{tabular}{|l|l|l|l|l|l|}
1 & 2 & 3 & 4 & 5
\end{tabular}

\begin{tabular}{|l|l|l|l|l|}
\hline 1 & 2 & 3 & 4 & 5
\end{tabular}

\begin{tabular}{|l|l|l|l|l|}
1 & 2 & 3 & 4 & 5 \\
\hline 1 & 2 & 3 & 4 & 5
\end{tabular}

\begin{tabular}{l|l|l|l|l}
1 & 2 & 3 & 4 & 5 \\
\hline 1 & 2 & 3 & 4 & 5
\end{tabular}

\begin{tabular}{ll|l|l|l|l}
1 & 2 & 3 & 4 & 5 \\
\hline 1 & 2 & 3 & 4 & 5
\end{tabular}

\begin{tabular}{ll|l|l|l|l}
1 & 2 & 3 & 4 & 5
\end{tabular}

\begin{tabular}{ll|l|l|l|l}
1 & 2 & 3 & 4 & 5 \\
\hline
\end{tabular}

\begin{tabular}{|l|l|l|l|l|}
1 & 2 & 3 & 4 & 5 \\
\hline
\end{tabular}

\begin{tabular}{l|l|l|l|l|l}
1 & 2 & 3 & 4 & 5
\end{tabular}

\begin{tabular}{llll|l|l} 
& & & & & \\
\hline 1 & 2 & 3 & 4 & 5
\end{tabular}

\begin{tabular}{ll|l|l|l|l|}
\hline & 2 & 3 & 4 & 5 \\
\hline 1 & 2 & 3 & 4 & 5 \\
\hline 1 & 2 & 3 & 4 & 5 \\
\hline
\end{tabular}


Indique as estratégias metodológicas de ensino que vocé utiliza em suas aulas:

Alio as tecnologias educacionais à(s) minha(s) disciplina(s).

Utilizo as tecnologias educacionais em sala de aula como material ilustrativo para determinados conteúdos.

Utilizo com mais frequência o quadro e gia do que as tecnologias educacionais.

Prefiro utilizar textos impressos às tecnologias educacionais.

Planejo aulas utilizando as tecnologias educacionais.

Planejo aulas em que os alunos necessitam do acesso constante à rede de internet.

Em minhas aulas solicito aos alunos a leitura prévia de materiais (livros, textos, artigos, etc.)

Minhas aulas são compostas de práticas do que foi previamente lido pelos alunos.

Propicio aos meus alunos momentos de atividades práticas após a apresentaçăo da teoria.

Em minhas aulas os alunos tentam encontrar a solução para um problema definido antecipadamente utilizando as tecnologias educacionais.

Utilizo a rotação por estações de aprendizagem em minhas aulas, em que os alunos vivenciam diferentes atividades de um mesmo conteúdo.

Utilizo aulas expositivas dialogadas.

Levo meus alunos ao laboratório de informática.

Utilizo as tecnologias em minhas aulas com uma perspectiva técnica, ou seja, para que o aluno se aproprie do computador de maneira a saber trabalhar com Word, Excel e outros aplicativos de editoração de textos.

Utilizo slides em sala de aula

Oportunizo aos alunos a produçăo de slides.

Utilizo a lousa digital em sala de aula.

Utilizo softwores educativos em sala de aula.

Permito que meus alunos utilizem notebooks em sala de aula.

Utilizo o celular em sala de aula para fins pedagógicos.

Permito que os alunos, no momento da aula, pesquisem informações em ambientes digitais sobre os conteúdos que estão sendo abordados.

Aproveito a expertise dos meus alunos com a utilização de tecnologias educacionais para contribuir em minhas aulas.

Elaboro, juntamente com alunos, projetos utilizando as tecnologias educacionais.

Meus alunos fazem apresentaçăo em grupo utilizando as tecnologias educacionais.

Propicio aos meus alunos momentos de produçăo de vídeos.

Utilizo filmes como recursos pedagógicos em minhas aulas.

Produzo vídeos para enriquecer minhas aulas.

Promovo aos alunos o acesso e uso das redes sociais.

Produzo áudio com os alunos em sala de aula.

Promovo a participação dos alunos em fóruns.

Promovo a participação dos alunos em chots.

Promovo aos alunos a ediçäo de fotos

Crio blogs com os meus alunos para fins pedagógicos.

Promovo atividades em que os alunos utilizem o ambiente virtual de aprendizagem (AVA) da IES em que leciono.

Possibilito diferentes estratégias metodológicas para a formação de meus alunos.

Possibilito momentos de reflexão teórica com os meus alunos sobre a utilização de tecnologia: educacionais.

Possibilito momentos de atividades práticas aos meus alunos que oportunizem a utilização de tecnologias educacionais. 


\begin{tabular}{|c|c|c|}
\hline INFRAESTRUTURA E INCENTIVO INSTITUCIONAL & $\underline{\Sigma}$ & $\frac{0}{12}$ \\
\hline \multicolumn{3}{|l|}{ Na Instituição de Ensino Superior que leciono há... } \\
\hline laboratório de informática em condiçôes de uso. & 1 & 2 \\
\hline computadores em quantidade suficiente para todos os alunos. & 1 & 2 \\
\hline computador em todas as salas de aula para utilização do(a) professor(a). & 1 & 2 \\
\hline wifi de boa qualidade liberada para todos os alunos. & 1 & 2 \\
\hline projetor multimidia em condiçäo de uso nas salas de aula. & 1 & 2 \\
\hline lousa digital em sala de aula. & 1 & 2 \\
\hline softwares educacionais para utilização nas aulas. & 1 & 2 \\
\hline suporte técnico para apoio na utilização de tecnologias educacionais em ala de aula. & 1 & 2 \\
\hline ambiente virtual de aprendizagem (AVA) para fins pedagógicos. & 1 & 2 \\
\hline cultura institucional para a utilizaçäo de tecnologias educacionais. & 1 & 2 \\
\hline $\begin{array}{l}\text { troca de experiéncias com os demais colegas (professores universitários) sobre o uso de } \\
\text { tecnologias educacionais. }\end{array}$ & 1 & 2 \\
\hline $\begin{array}{l}\text { Projeto Pedagógico do Curso contendo orientaçôes para a utilização de tecnologias } \\
\text { educacionais. }\end{array}$ & 1 & 2 \\
\hline $\begin{array}{l}\text { apoio por parte da equipe pedagógica de maneira a incentivar a utilização de tecnologias } \\
\text { educacionais nas aulas. }\end{array}$ & 1 & 2 \\
\hline $\begin{array}{l}\text { acompanhamento de minha prática docente no intuito de verificar se as tecnologias } \\
\text { educacionais estão sendo utilizadas em sala de aula. }\end{array}$ & 1 & 2 \\
\hline incentivo da direção académica para a utilização de tecnologias educacionais nas aulas. & 1 & 2 \\
\hline $\begin{array}{l}\text { participação do corpo docente na escolha e seleç̃̃o de tecnologias educacionais a serem } \\
\text { adquiridas pela IES. }\end{array}$ & 1 & 2 \\
\hline
\end{tabular}

\begin{tabular}{|c|c|c|c|c|}
\hline FORMAÇÃO INICIAL PARA A UTILIZAÇÃO DE TECNOLOGIAS EDUCACIONAIS & $\frac{\$}{\frac{5}{2}}$ & $\begin{array}{l}\hat{4} \\
\frac{1}{4}\end{array}$ & 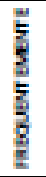 & $\frac{5}{\frac{2}{2}}$ \\
\hline $\begin{array}{l}\text { Em minha formação inicial (graduação) cursei disciplina(s) que abordava(m) a temática "Educação e } \\
\text { Tecnologias". }\end{array}$ & 1 & 2 & 3 & 4 \\
\hline $\begin{array}{l}\text { Minha formação inicial (graduação) ofereceu aporte teórico a fim de refletir sobre a utilização de } \\
\text { tecnologias educacionais. }\end{array}$ & 1 & 2 & 3 & 4 \\
\hline $\begin{array}{l}\text { Minha formaçâo inicial (graduaçâo) ofereceu momentos de prática sobre como utilizar as tecnologias } \\
\text { educacionais em sala de aula. }\end{array}$ & 1 & 2 & 3 & 4 \\
\hline $\begin{array}{l}\text { Em minha formação inicial (graduação) cursei disciplina(s) que me preparou(aram) para utilizar as } \\
\text { tecnologias em "sala de aula com os alunos". }\end{array}$ & 1 & 2 & 3 & 4 \\
\hline $\begin{array}{l}\text { Em minha formação inicial (graduação) a tecnologia foi abordada em uma perspectiva de uso pessoal e } \\
\text { näo para o uso didático. }\end{array}$ & 1 & 2 & 3 & 4 \\
\hline $\begin{array}{l}\text { Grande parte de minha aprendizagem com as tecnologias educacionais se deve a observação de bons } \\
\text { professores durante o tempo que passei na graduação. }\end{array}$ & 1 & 2 & 3 & 4 \\
\hline
\end{tabular}




\begin{tabular}{|c|c|c|c|c|}
\hline FORMAÇĂOO CONTINUADA PARA A UTILIZAÇĂOO DE TECNOLOGIAS EDUCACIONAIS & $\frac{\$}{\frac{\$}{2}}$ & $\begin{array}{l}\hat{4} \\
\frac{8}{2}\end{array}$ & 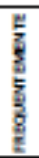 & $\frac{\bar{k}}{\tilde{k}}$ \\
\hline $\begin{array}{l}\text { A IES em que leciono proporciona cursos de formação continuada para utilização de tecnologias } \\
\text { educacionais em sala de aula. }\end{array}$ & 1 & 2 & 3 & 4 \\
\hline Participo de cursos sobre a utilização de tecnologjas educacionais ofertados pela IES que leciono. & 1 & 2 & 3 & 4 \\
\hline $\begin{array}{l}\text { Fiz ou faço cursos sobre a utilizaçăo de tecnologias educacionais além dos ofertados pela IES que } \\
\text { leciono. }\end{array}$ & 1 & 2 & 3 & 4 \\
\hline $\begin{array}{l}\text { A IES em que leciono oferta cursos de formação continuada para o uso de metodologias ativas } \\
\text { incorporando na formaçäo o uso de tecnologias educacionais associadas à metodologja. }\end{array}$ & 1 & 2 & 3 & 4 \\
\hline $\begin{array}{l}\text { A maior parte de minha aprendizagem com as tecnologias educacionais se deve a experiência no dia a dia } \\
\text { na sala de aula. }\end{array}$ & 1 & 2 & 3 & 4 \\
\hline Passei por uma preparação para a utilização de tecnologias educacionais em curso(s) de pós-ģraduação. & 1 & 2 & 3 & 4 \\
\hline Busco informações sobre as tecnologias educacionais autonomamente. & 1 & 2 & 3 & 4 \\
\hline Realizo pesquizas na área da "Educação e Tecnologias". & 1 & 2 & 3 & 4 \\
\hline
\end{tabular}

Indique em uma escala de 0 a 10 - sendo 0 a menor escala e 10 a maior - a importiancia que vocé atribui para a oferta de cursos de formação de professores:

Formação para a utilização de tecnologias educacionais no processo ensino-aprendizagem

Formaçäo teórica sobre as tecnologias educacionais

Formação para o desenvolvimento de novas estratégjas metodológicas 